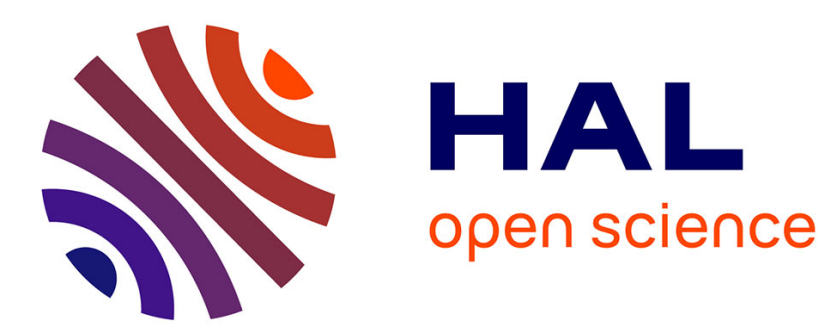

\title{
Evidence for a cost of time in the invigoration of isometric reaching movements
}

B. Berret, Gabriel Baud-Bovy

\section{To cite this version:}

B. Berret, Gabriel Baud-Bovy. Evidence for a cost of time in the invigoration of isometric reaching movements. 2022. hal-03475795v2

\section{HAL Id: hal-03475795 \\ https://hal.science/hal-03475795v2}

Preprint submitted on 24 Jan 2022

HAL is a multi-disciplinary open access archive for the deposit and dissemination of scientific research documents, whether they are published or not. The documents may come from teaching and research institutions in France or abroad, or from public or private research centers.
L'archive ouverte pluridisciplinaire $\mathbf{H A L}$, est destinée au dépôt et à la diffusion de documents scientifiques de niveau recherche, publiés ou non, émanant des établissements d'enseignement et de recherche français ou étrangers, des laboratoires publics ou privés. 


\title{
Evidence for a cost of time in the invigoration of isometric reaching movements
}

\author{
Bastien Berret ${ }^{*, 1,2,3}$ and Gabriel Baud-Bovy ${ }^{4,5}$ \\ ${ }^{1}$ Université Paris-Saclay CIAMS, 91405, Orsay, France \\ ${ }^{2}$ CIAMS, Université d'Orléans, 45067, Orléans, France \\ ${ }^{3}$ Institut Universitaire de France, Paris, France \\ ${ }^{4}$ Robotics, Brain and Cognitive Sciences Unit, Istituto Italiano di Tecnologia, Genoa, Italy \\ ${ }^{5}$ Faculty of Psychology, Vita-Salute San Raffaele University, Milan, Italy
}

\begin{abstract}
How the brain determines the vigor of goal-directed movements is a fundamental question in neuroscience. Recent evidence has suggested that vigor results from a trade-off between a cost related to movement production (cost of movement) and a cost related to our brain's tendency to temporally discount the value of future reward (cost of time). However, whether it is critical to hypothesize a cost of time to explain the vigor of basic reaching movements with intangible reward is unclear because the cost of movement may be theoretically sufficient for this purpose. Here we directly address this issue by designing an isometric reaching task whose completion can be accurate and effortless in prefixed durations. The cost of time hypothesis predicts that participants should be prone to spend energy to save time even if the task can be accomplished at virtually no motor cost. Accordingly, we found that all participants generated substantial amounts of force to invigorate task accomplishment, especially when the prefixed duration was long enough. Remarkably, the time saved by each participant was linked to their original vigor in the task and predicted by an optimal control model balancing out movement and time costs. Taken together, these results supports the existence of an idiosyncratic, cognitive cost of time that underlies the invigoration of basic isometric reaching movements.
\end{abstract}

\section{New and Noteworthy}

Movement vigor is generally thought to result from a trade-off between time and motor costs. However, it remains unclear whether the time cost only modulates vigor around some nominal value explained by a minimal motor cost or whether it determines movement invigoration more broadly. Here, we present an original paradigm allowing to neutralize the cost of movement, and provide new evidence that a cost of time must underlie the invigoration of isometric reaching movements.

\section{Introduction}

Understanding the principles underlying movement invigoration is an important topic in neuroscience given its potential implications for disorders such as Parkinson's disease (Mazzoni et al., 2007). Parkinsonian patients typically suffer from bradykinesia (Berardelli et al., 2001), an overall movement slowness which is related to a dysfunction of the basal ganglia (Turner and Desmurget, 2010; Dudman and Krakauer, 2016; Robbe and Dudman, 2020). Vigor generally characterizes the preferred speed of movement, which has been experimentally quantified through the -idiosyncraticincrease of velocity and duration as a function of distance in simple point-to-point movements. Large differences in vigor were reported across individuals but, remarkably, these differences were

\footnotetext{
*Corresponding author: bastien.berret@universite-paris-saclay.fr
} 
consistent between/within sessions or between movement modalities (e.g. head vs hand movements, dominant vs non-dominant reaching or vs walking Reppert et al., 2018; Berret et al., 2018; Labaune et al., 2020). While biomechanical factors may of course play a role, inter-individual differences of vigor were primarily attributed to psycho-economical processes that reflect a trait-like feature of individuality (Shadmehr et al., 2016; Berret et al., 2018; Shadmehr et al., 2019; Carland et al., 2019; Shadmehr and Ahmed, 2020a). The main results point to a cost of time related to the brain's tendency to discount the value of delayed rewards (Shadmehr et al., 2010; Haith et al., 2012; Rigoux and Guigon, 2012; Choi et al., 2014; Berret and Jean, 2016). Assuming that the goal of any movement is to put the system in a more rewarding state (Shadmehr, 2010), moving slowly would decrease the subjective value of reward and would thus be avoided in the control of goal-directed actions. This principle has been formalized in optimal control theory by using a cost of time that explicitly penalizes the passage of time (Hoff, 1994; Berret and Jean, 2016), thereby compensating for the inability of classical trajectory-formation models to account for the duration of self-paced movement (e.g. Flash and Hogan, 1985; Uno et al., 1989; Berret et al., 2011). Together with other model-based studies linking vigor to a global background signal representing the opportunity cost of time (average rate of reward or capture rate, Niv et al., 2007; Yoon et al., 2018), this suggests that a time cost could be the cornerstone of movement invigoration (Fig. 1A).

However, a cost of movement alone may be theoretically sufficient to explain our preferred speed (Fig. 1B). Objective measures of the cost of movement have shown that metabolic energy expenditure increases at both low and fast speeds, which is a well-known result for walking that seems to be valid for arm reaching as well (Ralston, 1958; Huang and Ahmed, 2012; Shadmehr et al., 2016). Furthermore, people may be reluctant to move slowly for physiological reasons related to the properties of the underlying control system (Park et al., 2017; Guigon et al., 2019). Endpoint variance tends to increase at both fast and slow speeds due to the effects of signal-dependent and constant motor noise unless efficient feedback corrections are triggered by the central nervous system (Wang et al., 2016). Effort and variance are two fundamental features of sensorimotor control that are thought to compose the cost of movement (Todorov and Jordan, 2002; Liu and Todorov, 2007; Gaveau et al., 2014, 2021; Berret and Jean, 2020). Interestingly, using a cost of movement was sufficient to explain the speed/accuracy trade-off-Fitts' law- (Harris and Wolpert, 1998; Qian et al., 2013) and the increase of velocity and duration as a function of distance in point-to-point movements from mechanistic reasons only (van Beers, 2008; Berret et al., 2021). In this case, existing inter-individual differences of vigor would result from discrepancies in the subjective cost of movement represented by people and biomechanical factors, and the cost of time would explain changes of vigor around a certain nominal value in response to modulation of reward or urgency (Takikawa et al., 2002; Reppert et al., 2015; Manohar et al., 2015; Thura, 2020). Yet, it has been recently suggested that the cost of movement could be itself discounted over time (Shadmehr et al., 2016; Summerside et al., 2018) such that movement vigor would essentially reveals "how much we value the things we are moving toward" (Shadmehr and Ahmed, 2020a,b). Because of this uncertainty about the cost of movement actually represented by subjects, the role played by the cost of time in daily actions with intangible rewards remains putative. Hence, does the cost of time mainly serve to modulate vigor around some nominal value defined by a minimum movement cost, or does it broadly set movement invigoration?

The goal of the present study was to clarify this issue by neutralizing the cost of movement experimentally. To this aim, we designed an isometric pointing task whose completion could be accurate and effortless in prefixed durations. This is like an airport treadmill that carries travelers to their destination in more or less time depending on whether they stand still or walk on it. Here participants had to control a cursor on a screen via a force applied onto an isometric joystick. A baseline condition was first tested to characterize the behavior of participants in such an isometric reaching task where the reward -if any- is intangible, and estimate their original vigor. In a second condition, the cursor was moved by an external drive such that the task could be accomplished successfully in a certain duration without any participant intervention. If time matters in the control of this isometric reaching task, we predicted that the participants should exert a substantial amount of force onto the joystick to save time, especially when the drive duration 

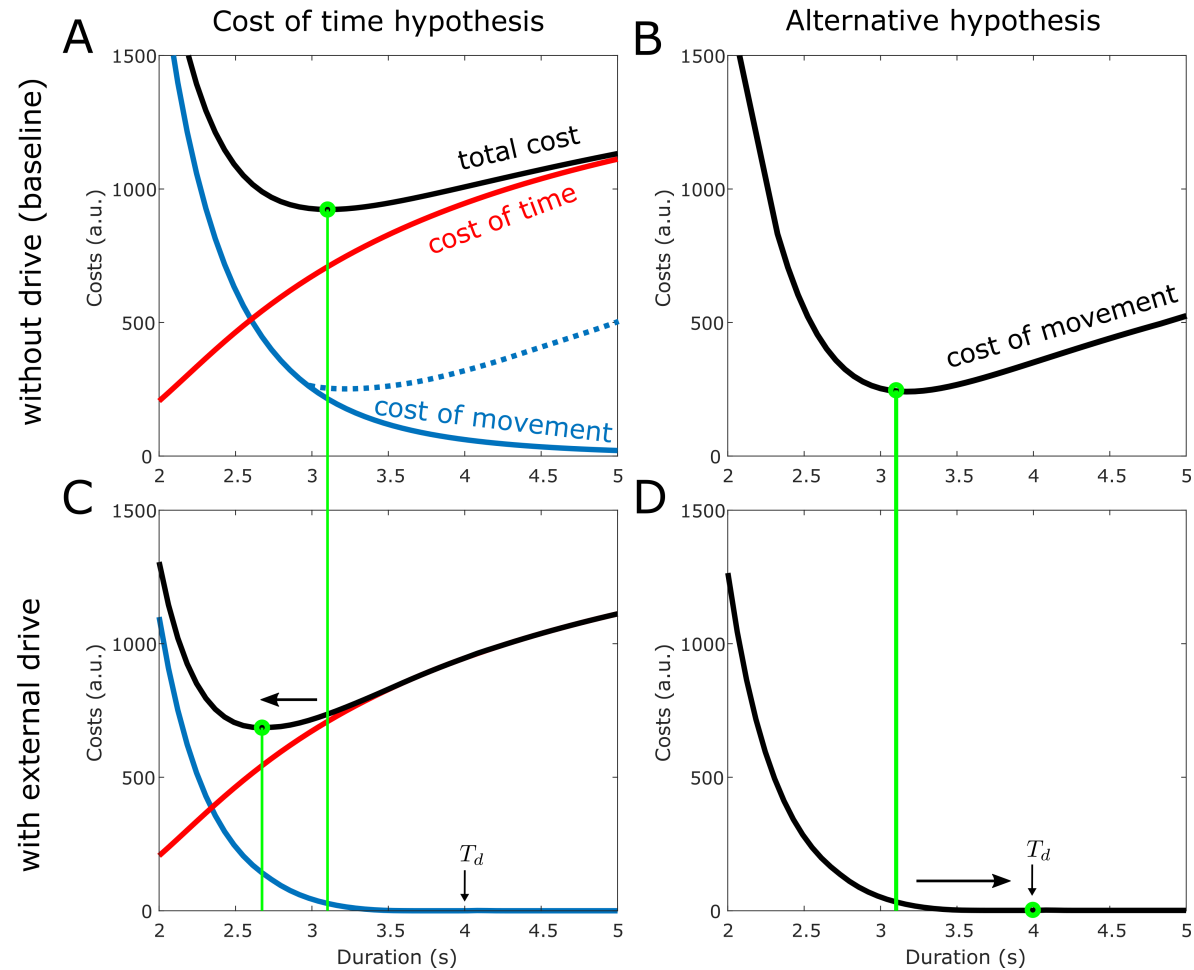

Figure 1: Vigor prediction for the different hypotheses. A. Prediction of vigor within the cost of time hypothesis. The preferred duration of a given point-to-point movement (here $3.1 \mathrm{~s}$ ) is obtained as a trade-off between a cost of movement (blue trace) and a cost of time (red trace). Note that the cost of movement could possibly increase for longer durations (dotted blue trace). B. Prediction of vigor using the cost of movement alone (alternative hypothesis). The preferred duration is the same and the two hypotheses are indistinguishable in this baseline condition. C. Prediction of movement vigor within the cost of time hypothesis in presence of an external drive. The assisting drive (here executing the task in duration $T_{d}=4 \mathrm{~s}$ ) reshapes the cost of movement such that the task can be accomplished with a smaller movement cost for durations shorter than the drive duration, and at no movement cost for longer durations. Due to the drive and the cost of time, the preferred duration will be shorter than the initial duration (here $2.7 \mathrm{~s}$ ). D. Prediction of vigor using a cost of movement alone in presence of an external drive. Again, the drive reshapes the cost of movement so that the preferred duration will be that of the drive because increasing speed would result in energy expenditure anyway. In this scenario, the preferred duration will be longer than the initial duration (here $4 \mathrm{~s}$ ). This illustrates that the two hypotheses make distinct predictions depending on whether the cost of time plays a predominant role in the invigoration of reaching actions without tangible reward. 
is long enough according to the cost of time hypothesis (Fig. 1C). Furthermore the time savings of each participant should be related to their original vigor in the task if it reflects how they implicitly value task accomplishment. In contrast, if time does not matter and vigor is essentially determined by a cost of movement in this task, participants should favor restful strategies even for a relatively long drive duration (Fig. 1D). Indeed, this would be the optimal behavior with respect to the minimization of any cost of movement, whether related to energy, inaccuracy or other factors.

\section{Materials and Methods}

\section{Participants}

The experiment included 15 participants ( 7 females, mean age \pm SD: $27 \pm 7$ ). All participants were contacted through a mailing list of persons who volunteer to do experiments at the Italian Institute of Technology and were paid 10 euros for the participation. All participant were naive with respect to the goal of the experiment and signed a written informed consent. The experimental protocol was approved by the local ethical committee (C.E.R. Liguria, P.R.063REG2016).

\section{Experimental methods}

Experimental apparatus The experimental setup comprised an isometric joystick that measured the force applied by the participant on it with a 6-dof force/torque sensor (Mini 45, ATI) and a screen (dimension $19 \mathrm{inch}$ ) in front of the participant (distance $65 \mathrm{~cm}$ ). The joystick was connected to a computer via a digital acquisition card (USB-6211, National Instrument). The force was sampled at $10 \mathrm{kHz}$ and filtered on-line by fitting a linear regression line to the most recent 160 samples. A custom $\mathrm{C}++$ program simulated the cursor dynamics at $1 \mathrm{kHz}$ and updated its position on the screen at $60 \mathrm{~Hz}$. The force and system state (position, velocity and external drive) were saved at $60 \mathrm{~Hz}$.

Task The participant sat in front of the table with the experimental setup and grasped the joystick with the right hand and the elbow bent at 90 degree angle. The goal of the task was to move a cursor from its home position to the target displayed on the screen by pushing the isometric joystick (see Fig. 2). Importantly, participants were always instructed to move the cursor at their preferred velocity. For simplicity the cursor was restricted to move only along the $y$-axis (bottom-up axis on the screen).

The experiment was divided into two sessions that were executed one after the other on the same day. The first experimental session (Session 1) served as a baseline condition from which the vigor and the cost of time of each participant were inferred. This session included four movement distances and no assistance was provided (amplitude 0.1, 0.2, 0.3 and 0.4 of screen size [height: $25.5 \mathrm{~cm}]$ ). The goal of this session was to find out the movement time corresponding to each amplitude, compute nominal vigor scores for each participant and identify the cost of time that would predict these movement times, given a cost of movement based on effort (Berret and Jean, 2016). There were 20 repetitions for each amplitude, thus yielding 80 trials in this session. The order of presentation of movement amplitudes was randomized. The second session (Session 2) included two movement distances (0.2 and 0.4). Importantly in this session, an external drive that generated biological movements reaching the target in different durations $\left(T_{d}=1,2,4\right.$ or $\left.6 \mathrm{~s}\right)$ was added to the cursor dynamics (see next paragraph).

An illustration of a trial from the participant's perspective is given in Figure 2. Each trial started with the display of the target and the initial cursor's position. The participants freely initiated the force and trajectory recording, and in the second session the external drive, by pushing the space bar. They were told to avoid pushing the joystick before this event. The self-starting of the recording was necessary in the second session to start the external drive and allow strategies that did not require the participant to push the joystick at all. The motion and recording terminated when the target was reached according to position and velocity thresholds defined below. The 


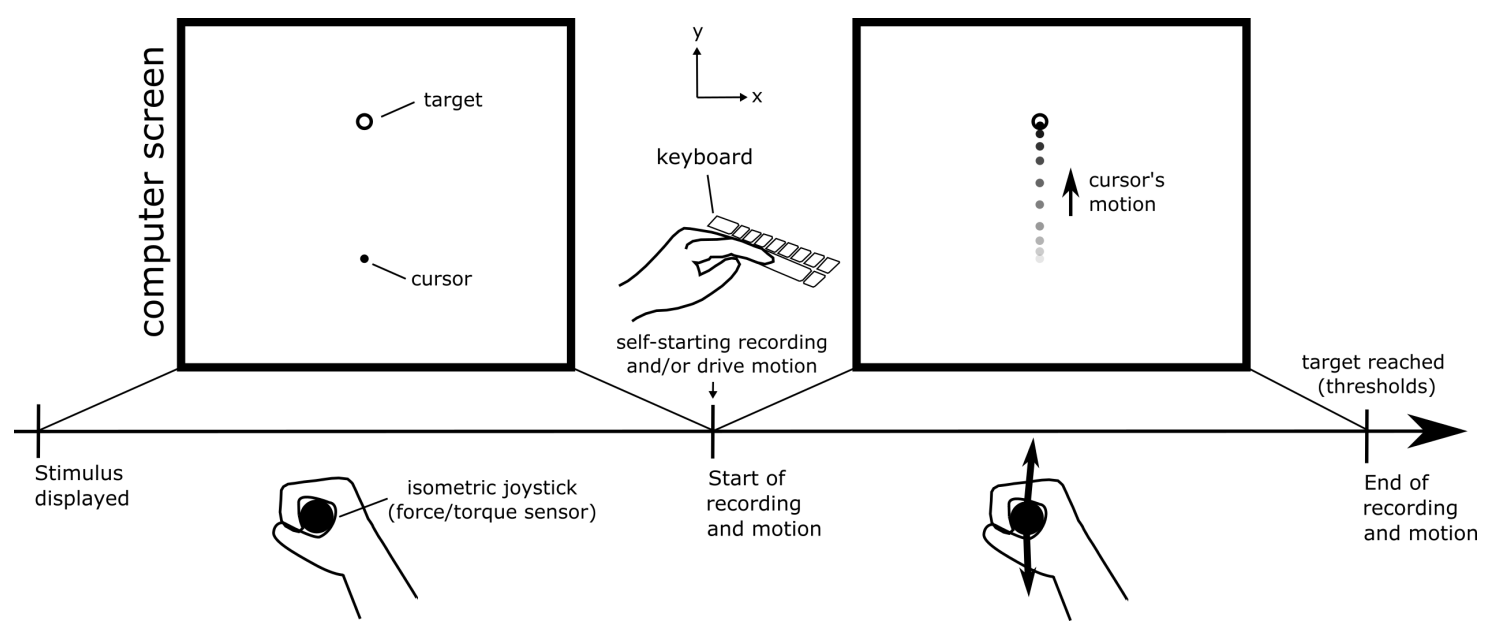

Figure 2: Trial structure from the participant's perspective. Each trial started with the display of the target on the screen and an invariant initial cursor's position. By pressing the space bar, the participant self-started the recording of the data and made it possible to accelerate the cursor by applying forces on the isometric joystick. In Session 2, this event also started the drive and so the cursor's displacement. The motion and recording terminated when the target was reached. The next trial with a new target occurred after a few seconds.

participant could start the next trial after completing the previous movement only if 3.5 seconds had elapsed from the beginning of the previous trial in order to limit the pace of the experiment when there was a strong external drive which led to very short trials. In Session 2, participants were aware of the presence and characteristics of the drive because each block started with two trials (one per amplitude) with a message on the screen instructing them to watch the cursor being moved to the target by the external drive in duration $T_{d}$. In this session, the participants were still instructed to move the cursor at their preferred velocity and to avoid pushing the joystick before pressing the space bar. There were 20 repetitions for each condition of amplitude and drive, thus yielding a total of 160 trials in this session. The task was executed from the slowest to the fastest drive with the amplitude randomized within each block. In Session 2, we used a block-wise design to ensure that the participant was fully aware of the characteristics of the drive. The participants were informed about this block-wise design without being told that the assistance will shift from slow to fast.

Cursor Dynamics The cursor dynamics along the $y$-axis was as follows:

$$
m \ddot{y}=f_{\mathrm{s}}-v \dot{y}
$$

where $m=25 \mathrm{~kg}$ and $v=10 \mathrm{~kg} / \mathrm{s}$ are the simulated mass and damping of the virtual system. These values were chosen during preliminary experiments to induce comfortable behaviors and force levels.

The force $f_{s}$ (measured by the force sensor on the y-axis) was the external force applied by the participant on the cursor. Hence, the force applied by the participant directly affected the cursor acceleration. The goal of the task in Session 1 was to move the cursor to a target $(1.275 \mathrm{~cm}$ width, that is, 0.05 of screen's height), $y_{f}$, from a given home position, $y_{0}$, at the participant's preferred speed.

Choice of external drive When an external drive was added, the cursor's dynamics was modified as:

$$
m \ddot{y}=f_{d}+f_{s}-v \dot{y}
$$

where $f_{d}$ is the external action implemented by an external controller. 
We noted in preliminary data that participants normally executed this isometric reaching task using bell-shaped velocity profiles. Therefore, we implemented an external drive as an optimal feedback control law that allowed to reach the target in a desired duration, $T_{d}$, with bell-shaped velocity profiles.

To this aim, we considered a minimum force change model as it predicts smooth, bell-shaped velocity profiles (Uno et al., 1989). We noted this optimal control $u_{d}$ which was the solution of the optimal control problem described hereafter. We denote by $\mathbf{x}=\left(y, \dot{y}, f_{d}\right)$ the column state vector and consider the dynamics:

$$
\dot{\mathbf{x}}=A \mathbf{x}+B_{d} u_{d}
$$

where

$$
A=\left(\begin{array}{ccc}
0 & 1 & 0 \\
0 & -v / m & 1 / m \\
0 & 0 & 0
\end{array}\right) \quad \text { and } \quad B_{d}=\left(\begin{array}{l}
0 \\
0 \\
1
\end{array}\right)
$$

The optimal drive was chosen to move the system from an initial state $\mathbf{x}_{0}=\left(y_{f}-y_{0}, 0,0\right)$ to a final state $\mathbf{x}_{f}=(0,0,0)$ in desired time $T_{d}$ while minimizing the cost:

$$
C\left(u_{d}\right)=\mathbf{x}\left(T_{d}\right)^{\top} Q_{f} \mathbf{x}\left(T_{d}\right)+\int_{0}^{T_{d}} u_{d}^{2}(t) \mathrm{d} t
$$

where $Q_{f}$ is the terminal cost matrix chosen as $Q_{f}=\operatorname{diag}\left(6.297 \times 10^{6}, 6.301 \times 10^{6}, 10\right)$. These parameters were also determined from preliminary simulations and tests to design an optimal drive that achieves the task reliably for all durations.

Solving this optimal control problem (linear-quadratic in finite horizon) yielded an optimal feedback control of the form (e.g. Stengel, 1986):

$$
u_{d}(t, \mathbf{x})=K(t) \mathbf{x}
$$

where $K(t)$ is the optimal feedback gain defined from $t=0$ to $t=T_{d}$. For $t \geq T_{d}$, we defined $K(t)=K\left(T_{d}\right)$. Note however that this situation was assumed not to occur in practice as we did not expect participants to move slower than the duration given by the external drive (it would be non-optimal with respect to both the cost of movement and the cost of time). We computed optimal control for drives with duration of $T_{d}$ equal to $1 \mathrm{~s}, 2 \mathrm{~s}, 4 \mathrm{~s}$ and $6 \mathrm{~s}$ and for distances equal to $y_{f}-y_{0}=0.2 \mathrm{~m}$ and $0.4 \mathrm{~m}$. Note that for simplicity we mapped the distances relative to screen size (see above) to S.I. units in the model for simulations (i.e. using the same numbers).

Coupled cursor dynamics Once the external drive is defined, we can consider the coupled dynamics used in the experiment of Session 2. Here the state-space dynamics was as follows:

$$
\dot{\mathrm{x}}=A \mathbf{x}+B_{d} u_{d}+B_{s} f_{s}
$$

where $B_{s}=(0,1 / m, 0)^{\top}$. The force input of the human participant thus affected directly the cursor acceleration.

\section{Data processing and parameters}

Kinematics The force was the only user input and was filtered on-line (see Experimental Apparatus). Position and velocity being integrated by the dynamic system (Eq. 6) were not filtered further. For each trial, we identified the beginning of the movement (velocity threshold $0.005 \mathrm{~m} / \mathrm{s}$ ). The trial and recording finished when the cursor position was inside the target (distance to its center less than $0.025 \mathrm{~m}$ ) with a velocity below $0.02 \mathrm{~m} / \mathrm{s}$. For each trial, we computed the movement duration and the movement amplitude. We also identified if a sub-movement was present (negative velocity) and counted the number of peaks in the force profile (each force peak corresponds to a period when the absolute of the force is above $1 \mathrm{~N}$ for at least $0.1 \mathrm{~s}$ ). We excluded from the analyses about $13 \%$ of the trials because there was movement reversal, more than five peaks in the force profile or the movement time differed from the condition average by more than three standard deviation. To compute average trajectories, single trial trajectories were resampled to have the same number of samples and average duration. 
Amount of force In order to estimate the effort produced by the participant using an objective measure, we considered the amount of force applied during the trial, defined as (Shadmehr et al., 2016):

$$
\int_{0}^{T_{s}}\left|f_{s}(t)\right| \mathrm{d} t
$$

Vigor scores In this paper we computed vigor scores using the maximum likelihood approach described by Reppert et al. (2018) but we used the duration of movements instead of peak velocities.

To this end, we first computed the grand mean duration of movements of each amplitude $A_{i} \in$ $\{0.1,0.2,0.3,0.4\}$ (data of all participants pooled together). These movement durations were then used to fit the affine function

$$
T_{i}=g\left(A_{i}\right)=\alpha+\beta A_{i}, \quad \alpha, \beta \in \mathbb{R}
$$

Then, we expressed the movement duration for each amplitude of each participant $j=1, \ldots, 15$ as:

$$
T_{i, j}=\frac{1}{\operatorname{vg}_{j}} g\left(A_{i}\right)+\varepsilon_{j}
$$

where $\varepsilon_{j} \sim \mathcal{N}\left(0, \sigma_{j}^{2}\right)$ (Gaussian variable with zero mean and variance $\sigma_{j}^{2}$ ). The quantity $\operatorname{vg}_{j}$ will denote the vigor score of participant $j$. A score of vigor greater than 1 will thus indicate a participant that is more vigorous than the average of the participants and therefore exhibits shorter movement durations in general.

Considering the log-likelihood of all the data points given the model parameters $\left\{\operatorname{vg}_{j}, \sigma_{j}^{2}\right\}_{j=1, \ldots, 15}$ and maximizing it with respect to $\mathrm{vg}_{j}$, yields the following formula for each participant $j \in$ $\{1, \ldots, 15\}$ :

$$
\operatorname{vg}_{j}=\frac{\sum_{i=1}^{4} g\left(A_{i}\right)^{2}}{\sum_{i=1}^{4} T_{i, j} g\left(A_{i}\right)}
$$

where $A_{i}$ is the movement amplitude and $T_{i, j}$ the individual mean movement duration for that amplitude.

Note that we also tested a canonical function $g\left(A_{i}\right)$ including a concave term $g\left(A_{i}\right)=\alpha+\beta A_{i}+$ $\gamma \log _{2}\left(A_{i} / W+1\right)$ where $W$ is the target's width (see Berret et al., 2018; Young et al., 2009) but this resulted in vigor scores that were highly correlated with the present ones $(r>0.99)$. Furthermore, we could have defined vigor scores from peak velocities as Reppert et al. (2018) but, again, the vigor scores were strongly correlated with the present ones $(r>0.99)$.

\section{Statistical analyses}

We used repeated-measure ANOVAs to analyze movement duration and average force as a function of movement amplitude and, in Session 2, drive duration. Both factors were coded with polynomial contrasts to conduct a trend analysis. All statistical analyses were conducted in R (R Core Team, 2019) with afex (Singmann et al., 2019) and emmeans (Lenth, 2019) packages.

\section{Optimal control modelling}

Here we model the behavior of participants in the second session. We extended the system state $\mathbf{x}=\left(y, \dot{y}, f_{d}\right)$ to include the human contribution, as $\tilde{\mathbf{x}}=\left(\mathbf{x}, f_{s}\right)$, and considered the following augmented dynamics:

$$
\dot{\tilde{\mathbf{x}}}=\tilde{A} \tilde{\mathbf{x}}+\tilde{B}_{d} u_{d}+\tilde{B}_{s} u_{s}
$$

with $\tilde{B}_{d}=(0,0,1,0)^{\top}$ and $\tilde{B}_{s}=(0,0,0,1)^{\top}$ and

$$
\tilde{A}=\left(\begin{array}{cccc}
0 & 1 & 0 & 0 \\
0 & -v / m & 1 / m & 1 / m \\
0 & 0 & 0 & 0 \\
0 & 0 & 0 & 0
\end{array}\right) .
$$


where $u_{d}$ is the optimal drive and $u_{s}$ is the human motor command. In this model, the motor command $u_{s}$ is at the level of force change, which reflects the fact that human force varies smoothly due to the low-pass filtering property of muscle contraction. The motor optimal drive $u_{d}$ is also at the level of force change and affects the cursor dynamics via $f_{d}$. In absence of participant intervention (i.e., $u_{s} \equiv 0$ ), the system is equivalent to Eq. 3 and the cursor moves according to the previously defined optimal drive.

Here, we considered the following cost function:

$$
C\left(u_{s}\right)=\int_{0}^{T_{s}}\left(u_{s}^{2}(t)+h(t)\right) \mathrm{d} t .
$$

where $T_{s}$ is the movement duration, $h(t)$ is the cost of time of the participant, and $u_{s}^{2}(t)$ counts their effort input in the task. We finally added terminal constraints to capture how a trial was terminated in the experiment. We imposed that the final velocity and position were respectively below $0.02 \mathrm{~m} / \mathrm{s}$ and $0.025 \mathrm{~m}$ in accordance with the experimental conditions given above.

The use of the optimal control model involved two steps: (1) an identification step with the data of Session 1; and (2) a prediction step to compare with the data of Session 2.

In the identification step, we followed the methodology documented in Berret and Jean (2016) and Berret et al. (2018) to identify the term $h(t)$ for each participant using the data of Session 1 where movement duration $T_{s}$ for each amplitude was obtained from linear regression (see above), and external drive was absent ( $u_{d} \equiv 0$ in the above equations). The cost of time was then fitted to a generalized sigmoid function to get an analytical formula for $h(t)$ (see Berret et al., 2018 for more details details about the procedure). In the prediction step, we solved an optimal control problem in free time to predict movement duration $T_{s}$ and the user's input $f_{s}$. In this step, the external drive $u_{d}$ was as defined experimentally and the cost of time $h(t)$ was as identified during the identification step, all the other parameters being unchanged.

The procedure was repeated for each distance of Session $2(0.2 \mathrm{~m}$ and $0.4 \mathrm{~m})$, each $T_{d}(1 \mathrm{~s}, 2 \mathrm{~s}, 4 \mathrm{~s}$ and $6 \mathrm{~s}$ ) and each participant (since each participant will have a different cost of time given that their behaviors in Session 1 will differ).

This model predicts that participants who perform optimally will find a trade-off between a certain physical effort and a cost related to the passage of time. In other words, if there is a cost of time, they should spend some energy to save time even when there is an assisting drive that can achieve the task by itself. An illustration of the balance between effort and time was provided in Fig. 1. If there is no cost of time, participants should not intervene as it would be the optimal strategy with respect to any cost of movement (for instance effort or accuracy here).

\section{Results}

\section{Experimental results of Session 1 (without drive)}

Mean trajectories Here we describe the average self-paced behavior of the participants in absence of an external drive. The grand mean trajectories are reported in Figure 3 for the distances $0.2 \mathrm{~m}$ (Fig. 3A) and $0.4 \mathrm{~m}$ (Fig. 3B) respectively. Velocity profiles are approximately bell-shaped even though the experimental protocol stopped motion according to some threshold that is visible at the end of the velocity profiles. Force profiles resemble acceleration profiles and mainly consist of a positive phase (to accelerate the cursor) and a negative phase to decelerate it. Small initial force values indicate that the participants respected well the instruction of not pushing on the joystick before pressing the space bar (initial force $0.39 \pm 0.66 \mathrm{~N}$ ). The force profiles do not return to zero because of the criterion used to detect the end of a trial (see Methods). Substantial inter-individual differences can be noted for instance on velocity peaks or on the force used to perform the task. Repeated-measures ANOVA confirmed that movement duration $\left(\mathrm{F}_{3,42}=85.33, \eta_{\mathrm{G}}^{2}=.39, \mathrm{p}<.001\right)$ and force $\left(\mathrm{F}_{3,42}=93.93, \eta_{\mathrm{G}}^{2}=.36, \mathrm{p}<.001\right)$ varied across amplitudes. A trend analysis revealed that movement time and average force increased linearly with movement amplitude for the range of values included in the study. 

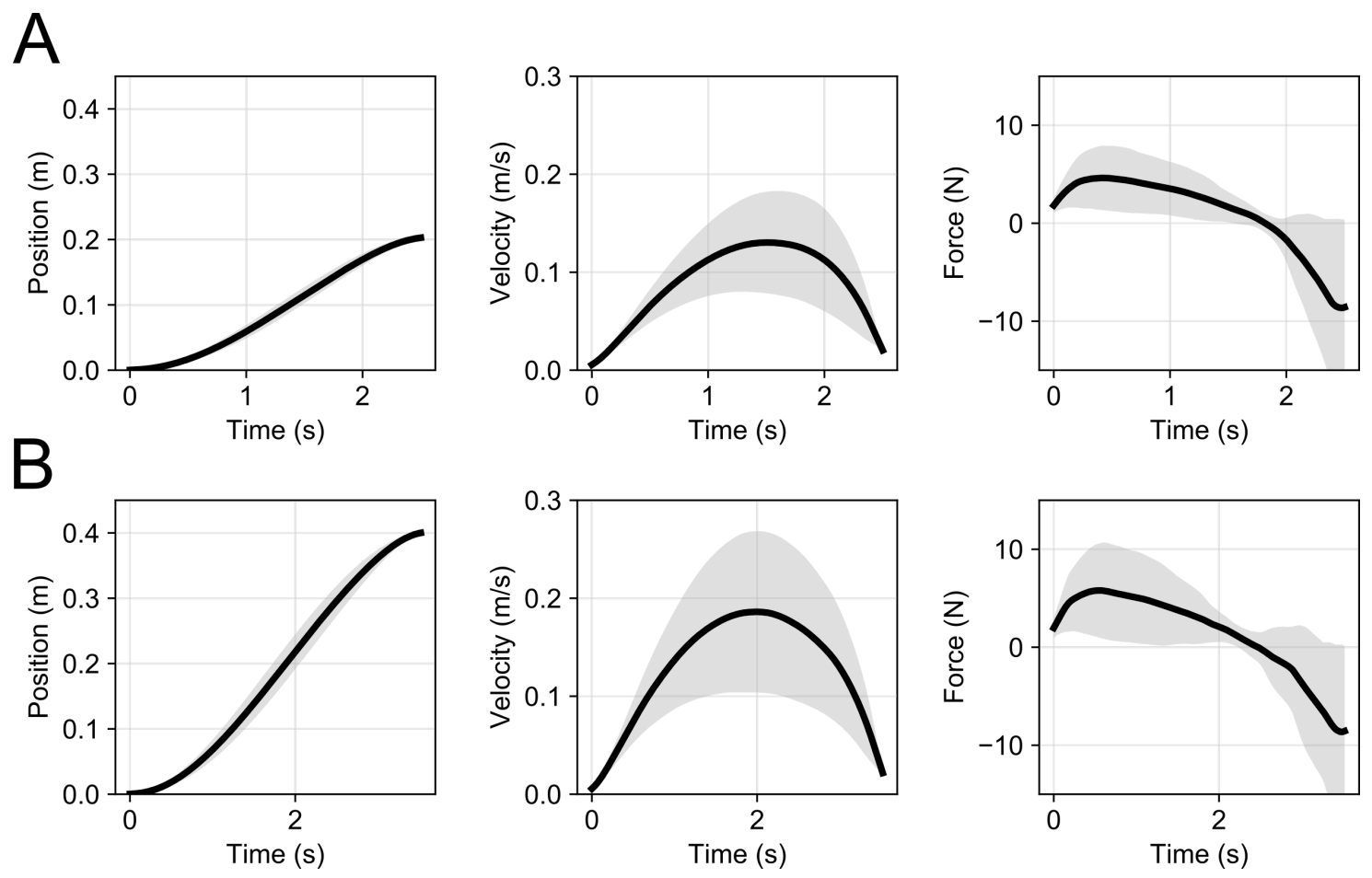

Figure 3: Grand mean trajectories in Session 1. A. Mean displacement, velocity and force for the distance $0.2 \mathrm{~m}$. Thick black lines are means across participants and shaded areas are standard deviations. Trajectories were normalized temporally before computing means and deviations. B. Same information for the distance $0.4 \mathrm{~m}$.

Amplitude-duration and amplitude-force relationships The overall behavior of participants in terms of movement duration and amount of force are shown in Figure 4 (black filled circles). As in real point-to-point arm reaching experiments, the duration of movement was found to increase with amplitude almost linearly, as confirmed by trend analysis (linear: $\mathrm{t}_{42}=15.88$, $\mathrm{p}<.001$; quadratic: $\left.\mathrm{t}_{42}=-1.95, \mathrm{p}=.06\right)$. Movements of distance corresponding to $0.2 \mathrm{~m}$ and $0.4 \mathrm{~m}$ were performed in about $2.5 \mathrm{~s}$ and $3.5 \mathrm{~s}$ respectively. The amount of force also increased with amplitude in a similar way (linear: $\mathrm{t}_{42}=16.76, \mathrm{p}<.001$; quadratic: $\mathrm{t}_{42}=-0.92, \mathrm{p}=0.36$ ). The grand mean amount of force was about $7 \mathrm{~N}$ for the distance $0.2 \mathrm{~m}$ and $11 \mathrm{~N}$ for the distance $0.4 \mathrm{~m}$. Again, a relatively large inter-individual variability was visible here and we thus focus on some individual performance scores in the following.

Individual movement vigor scores and costs of time The relationship between amplitude and duration for each individual is reported in Figure 5A. A color code is used to track each individual throughout the study. Linear regressions confirmed that duration increased with movement amplitude in an approximately affine way for every participant $\left(r^{2}=0.95 \pm 0.06\right.$, mean \pm std across participants). Using a fitting function including a $\log _{2}$ term, we got a slightly improved goodness of fit but with one additional parameter $\left(r^{2}=0.97 \pm 0.06\right)$. From these relationships, it was possible to compute vigor scores and sort individuals according to these scores (Fig. 5B) (see Methods).

Furthermore, the same relationships allowed us to identify the cost of time for each participant (see also Methods). The resultant costs of time are depicted in Figure 5C. As expected since both measures are derived from the same data, there is a strong correlation between the vigor of 

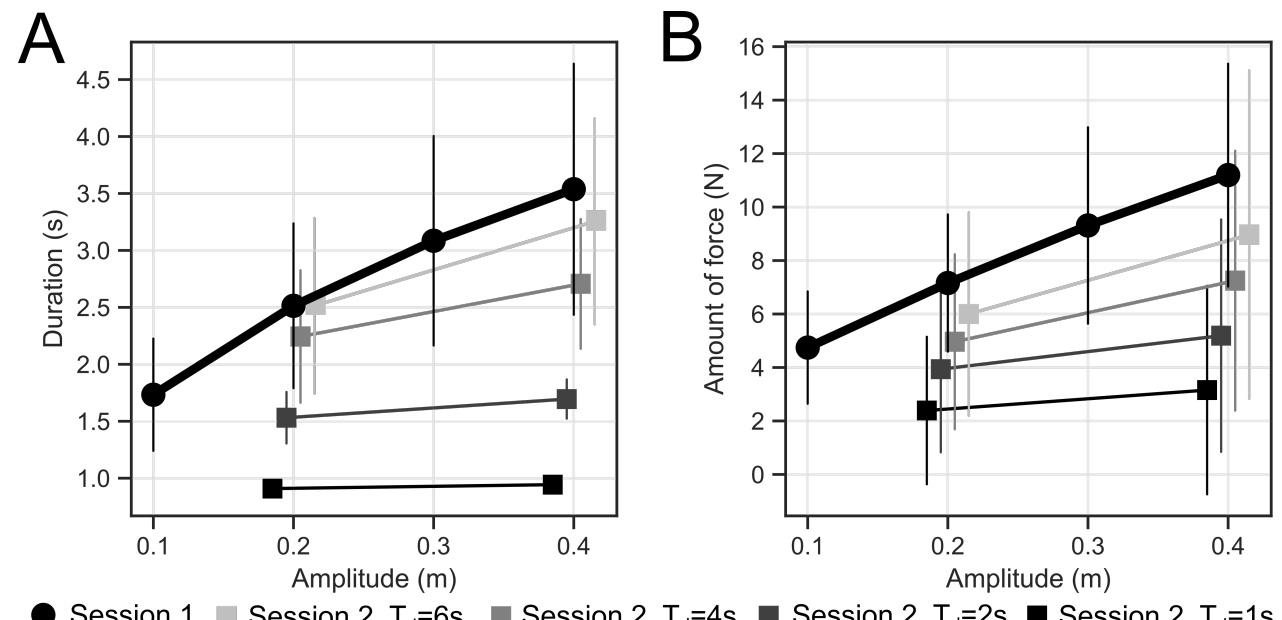

Figure 4: Mean behavior across participants as a function of distance. A. Amplitude-duration relationships in Sessions 1 and 2. Filled circles are data from Session 1 for the 4 tested amplitudes. Grey-colored filled squares are data from Session 2 for the 4 drives and the 2 amplitudes tested. Vertical bars represent standard deviations in all cases. B. Same information for the amplitudeamount of force relationships.

participants and the magnitude of their cost of time (Fig. 5D). Typically, the faster participants in Session 1 (i.e. with larger vigor scores) penalized time more steeply (i.e. increased more quickly, and to a higher asymptotic value, their time costs).

\section{Experimental and simulation results of Session 2 (with drive)}

We now consider the experimental data of Session 2 where the external drive allows, in theory, the participant to complete the task without any muscular force. Figure 6 depicts the grand mean behavior across participants for two different drives $(2 \mathrm{~s}$ and $6 \mathrm{~s})$ and the two distances tested in this Session $(0.2 \mathrm{~m}$ in panels A-C and $0.4 \mathrm{~m}$ in panels D-F). These two distances were illustrated for Session 1 in Fig. 3 for comparison. Black/grey traces show the experimental data and red/purple traces show the simulated data. The simulations relied on the costs of time identified from the data of Session 1 (one cost of time per participant) and the free-time optimal control simulations minimizing a trade-off between the cost of movement and the individual-based cost of time (which explains why simulated data also vary across participants). Velocity profiles are bell-shaped as in the first Session. Force profiles appear more irregular but they still present a positive and negative peaks that corresponds to an acceleration and deceleration of the cursor, even though the presence of the drive may both accelerate and decelerate the cursor on its own. The figure shows that participants used some force to save time, thus achieving the task in less than $3.5 \mathrm{~s}$ when the drive corresponded to a $6 \mathrm{~s}$ movement duration for example. Noticeably, participants still used some force on average even when the drive was stronger and would lead to a $2 \mathrm{~s}$ long movement on its own.

Mean movement duration and amounts of force across participants are reported in Figure 4 (filled squares). As expected, the performance became closer to that of Session 1 as the drive duration increased. Average movement duration increased with drive duration $\left(\mathrm{F}_{3,42}=69.25, \eta_{\mathrm{G}}^{2}=.68\right.$, $\mathrm{p}<.001)$ and amplitude $\left(\mathrm{F}_{1,14}=393.36, \eta_{\mathrm{G}}^{2}=.10, \mathrm{p}<.001\right)$. The statistically significant interaction $\left(\mathrm{F}_{3,42}=63.47, \eta_{\mathrm{G}}^{2}=.065, \mathrm{p}<.001\right)$ reflected a steeper increase of the movement duration with drive duration for large movement than for small movements (contrast between linear trends: $\mathrm{t}_{42}=16.62$, $\mathrm{p}<.001)$. A similar analysis of average force revealed the same trends. Average amount of force also increased with drive duration $\left(\mathrm{F}_{3,42}=17.27, \eta_{\mathrm{G}}^{2}=0.15, \mathrm{p}<.001\right)$ and amplitude $\left(\mathrm{F}_{1,14}=19.56\right.$, $\mathrm{p}<0.001)$. The statistically significant interaction $\left(\mathrm{F}_{3,42}=21.33, \eta_{\mathrm{G}}^{2}=0.01, \mathrm{p}<.001\right)$ reflected a steeper increase of the average force with drive duration for large movement than for small movements (contrast between linear trends: $\mathrm{t}_{42}=6.02, \mathrm{p}<.001$ ). 
A
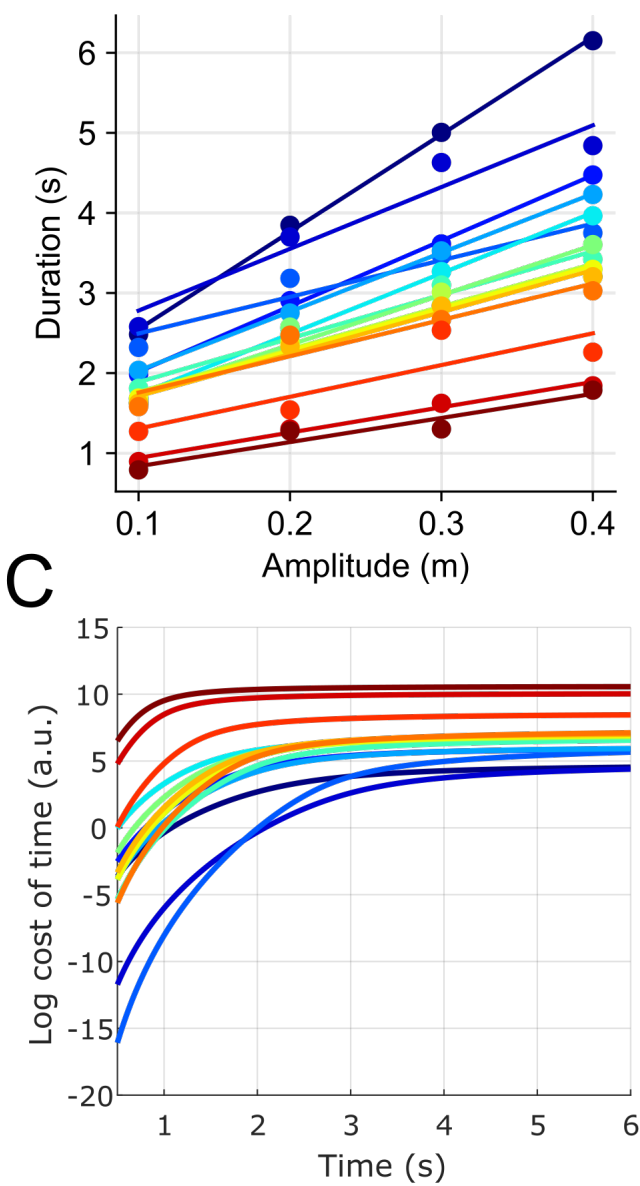

$\mathrm{B}$
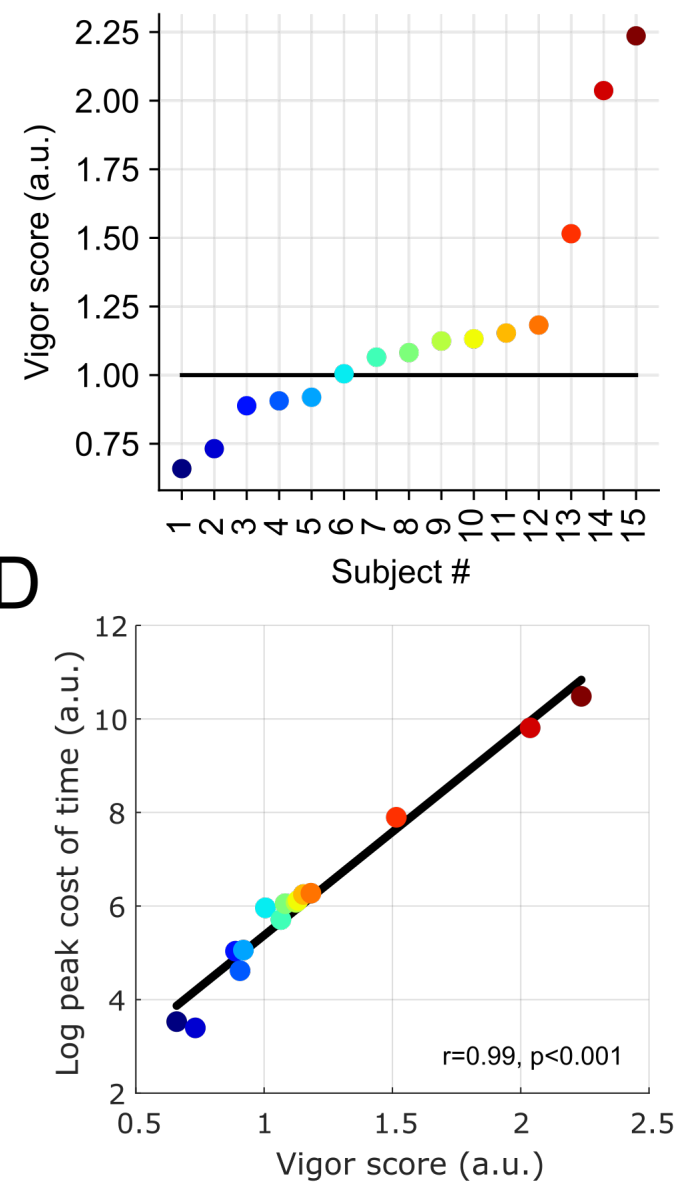

Figure 5: Individual vigor scores. A. Amplitude-duration relationships in Session 1. Each color is the data of one participant. Linear regression lines are displayed. The cost of time was inferred from these individual-level relationships. B. Corresponding vigor scores allowing to sort individuals from the slowest to the fastest in the isometric reaching task of Session 1. The horizontal black line corresponds to a reference vigor score of 1. C. Logarithm of the costs of time identified for each participant, i.e. $\log \left(\int_{0}^{T} h(t) \mathrm{d} t\right)$. The x-axis in this plot refers to the movement duration $T$. D. Correlation between the vigor scores and the logarithm of the peak derivative of the costs of time (i.e. maximal value of $h(t)$ ). 

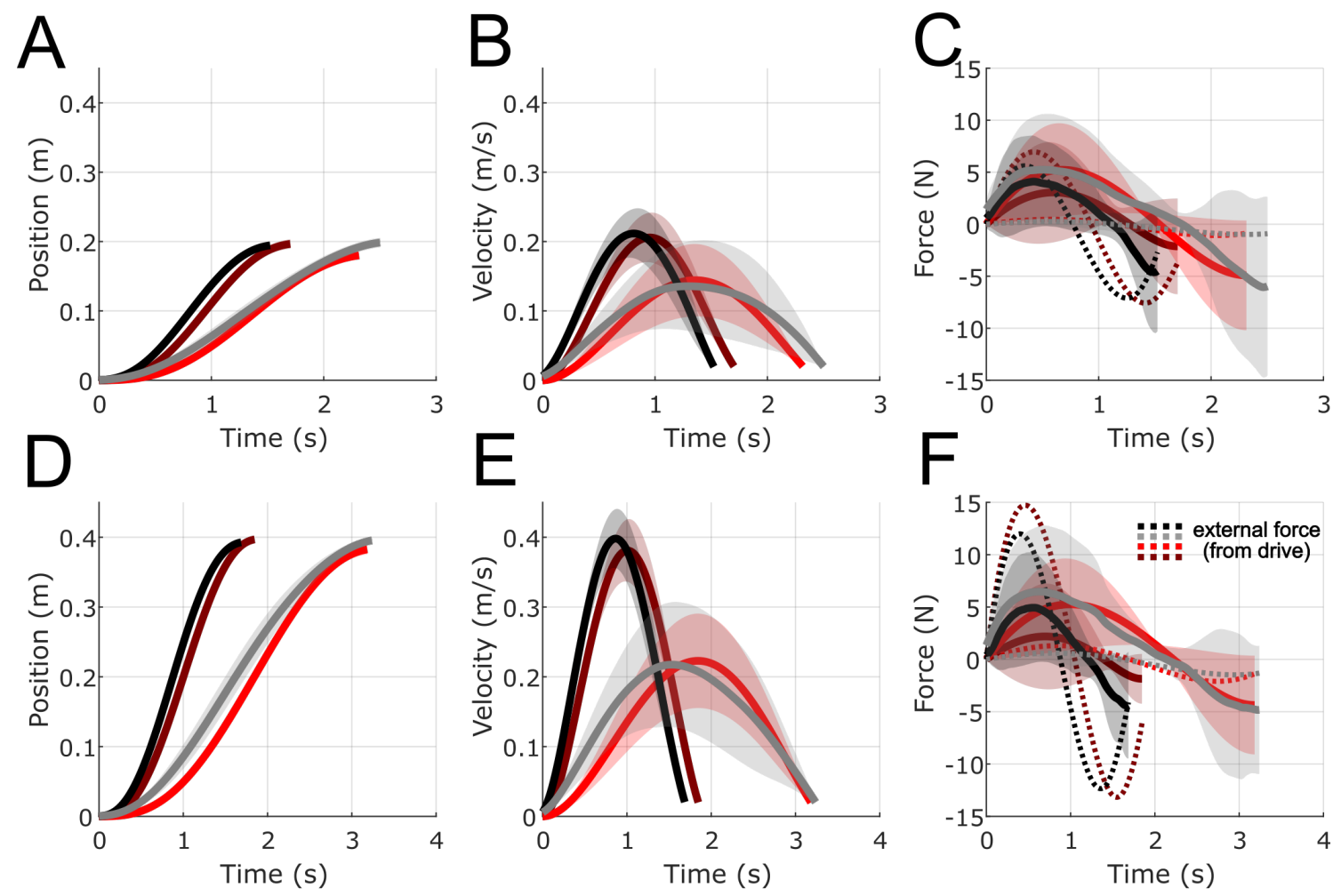

Exp. with $T_{d}=2 s$

Sim. with $T_{d}=2 s \quad$ Exp. with $T_{d}=6 s$

Sim. with $T_{d}=6 s$

Figure 6: Experimental and simulated trajectories in presence of the drive. A. Cursor displacements in the experiments (black/grey) and simulations (purple/red), for two different drive durations (2s and $6 \mathrm{~s}$ ), for the distance $0.2 \mathrm{~m}$. Mean trajectories across participants are reported with standard deviations as shaded areas. B. Same information for the velocity. C. Same information for the force. In solid line, the input force $f_{s}$ is depicted. In dotted line, the external force coming from the drive is also reported. D-F. Same information but for the distance $0.4 \mathrm{~m}$. 
In Figure 7, we depict mean movement durations chosen by participants in Session 2 and compare them to drive durations (black identity line -indicating the optimal duration if only a cost of movement is minimized-) and model predictions (red trace and shaded area). The overall behavior was as follows: movement duration increased with the duration of the drive and tended to plateau near the movement duration obtained in Session 1 for slow drives. Indeed, the movement duration of participants for a drive of $6 \mathrm{~s}$ was closed to the duration obtained in Session 1 (dotted line in Fig. 7). Noticeably, the model replicated quite well this average behavior. An optimal trade-off between movement and time costs thus leads to this evolution of movement duration with respect to drive duration for each distance.

Next, we analyzed the force that participants were prone to exert to save a given amount of time. We thus focused on the relationship between the gain of time and the amount of force used by the participants to complete the task, the gain of time being defined as the drive duration minus the actual duration. We found that all the participants tended to increase the amount of force to save more time across conditions (Fig. 8). The model replicated this trend quite well on average, especially for the longer drive durations. Important inter-individual differences could also be noted. For instance, to save one second, participants could use quite different amounts of force. In Fig. 8C for instance, participant \#1 (least vigorous) used less than $5 \mathrm{~N}$ to save 1 second while participant \#15 (most vigorous) used more than $10 \mathrm{~N}$ to save the same amount of time. It is worth noting that they saved the same amount of time for different drive durations $\left(T_{d}=4 \mathrm{~s}\right.$ for participant \#1 and $T_{d}=2 \mathrm{~s}$ for participant \#15). Our model captured these main inter-individual differences thanks to the subjective costs of time identified from the data of Session 1.

Finally, we investigated if there was any relationship between the vigor of participants in Session 1 and their behavior in Session 2 (Fig. 9). Remarkably, we found that the more vigorous participants in Session 1 were indeed the ones saving more time in Session 2 (high correlations with $\mathrm{r}>0.90$ for both tested distances, $\mathrm{N}=15$ participants). These participants were also the ones using a greater amount of force (correlation coefficients between vigor and average amount of force $r>0.90$-not depicted-). In summary, vigorous participants spent more energy to save more time.

\section{Discussion}

Recent studies have suggested that the vigor of movement results from a trade-off between a cost related to movement production and a cost related to the passage of time. However, whether a cost of time underlies the vigor of simple reaching movements remains unclear as a cost of movement could be theoretically sufficient to explain our preferred speed on average. This cost of time could mainly serve to modulate our speed around a reference value according to the urgency or reward of the task. To disambiguate this question, we designed an isometric pointing task that makes distinct predictions depending on whether a cost of movement or a cost of time is prevalent. In some conditions, an external drive moved the cursor to the target with a certain duration such that the task could be accomplished accurately and effortlessly with no participant intervention. Despite this self-completion of the task, the participants exerted all the more force the slower the movement of the cursor was and the higher their original vigor in the task was, in agreement with optimal control simulations assuming a trade-off between time and effort. These findings are compatible with the existence of an idiosyncratic, cognitive cost of time underlying the invigoration of basic isometric reaching movements.

Producing a movement inevitably entails a motor cost that can be translated into tangible variables such as metabolic energy, effort, smoothness, error or variance. The identification of the cost that best reproduces human trajectories has attracted a lot of attention in optimal control theory (see Engelbrecht, 2001; Todorov, 2004; Berret et al., 2019 for reviews) but it has proven difficult to 

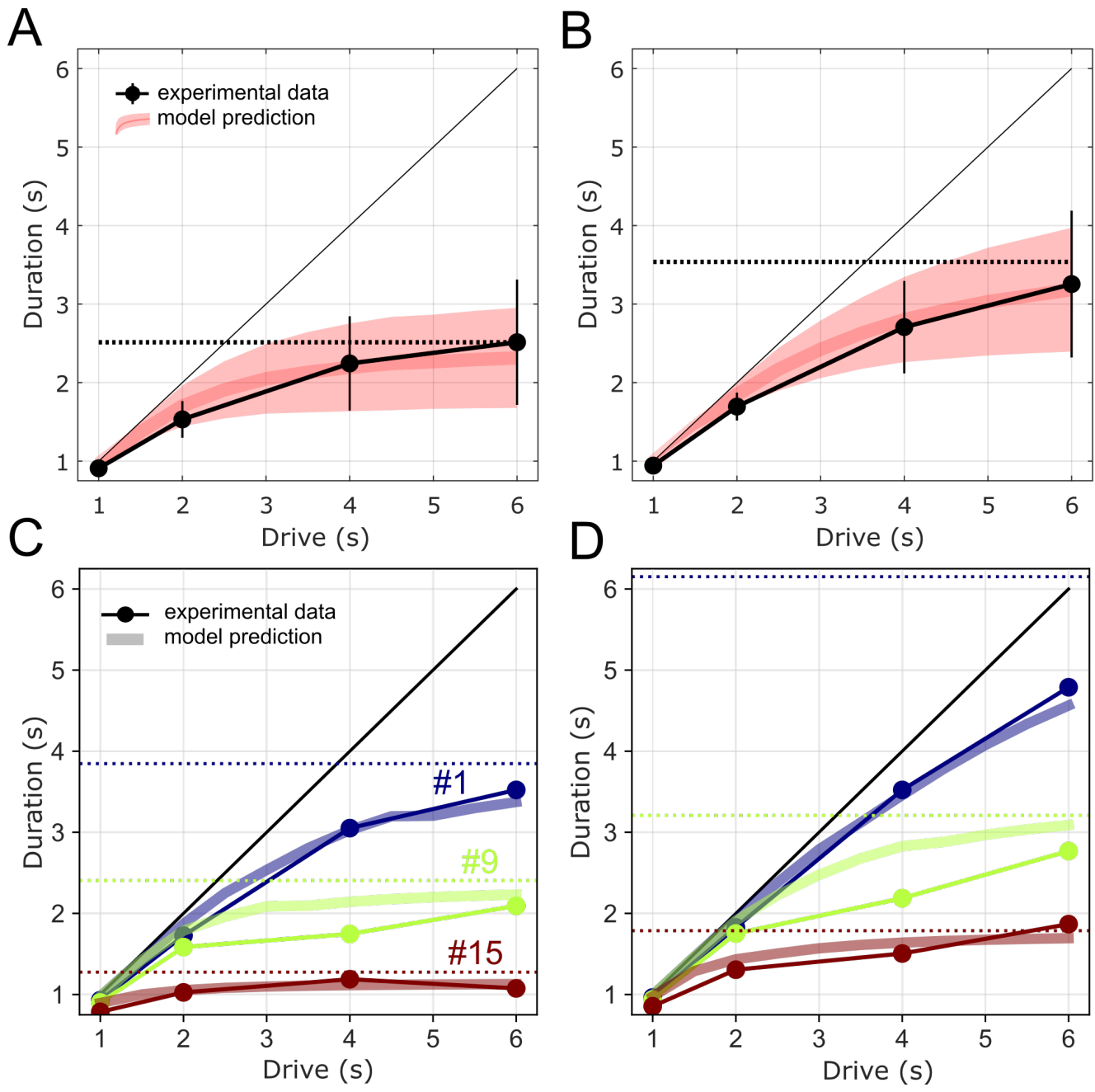

Figure 7: Movement duration with respect to drive duration in Session 2. A. Mean experimental and simulated data for distance $0.2 \mathrm{~m}$. Black markers and traces are experimental data. Mean and standard deviations across participants are reported. Red traces and shaded areas are model predictions (also mean and standard deviations across participants). The horizontal dotted line correspond to the mean duration measured in Session 1 for that distance. The identity line corresponds to the drive duration, which would the optimal duration if only a cost of movement is minimized. B. Same information for distance $0.4 \mathrm{~m}$. C-D. Same information for three participants with high, medium and low vigor respectively. 
A
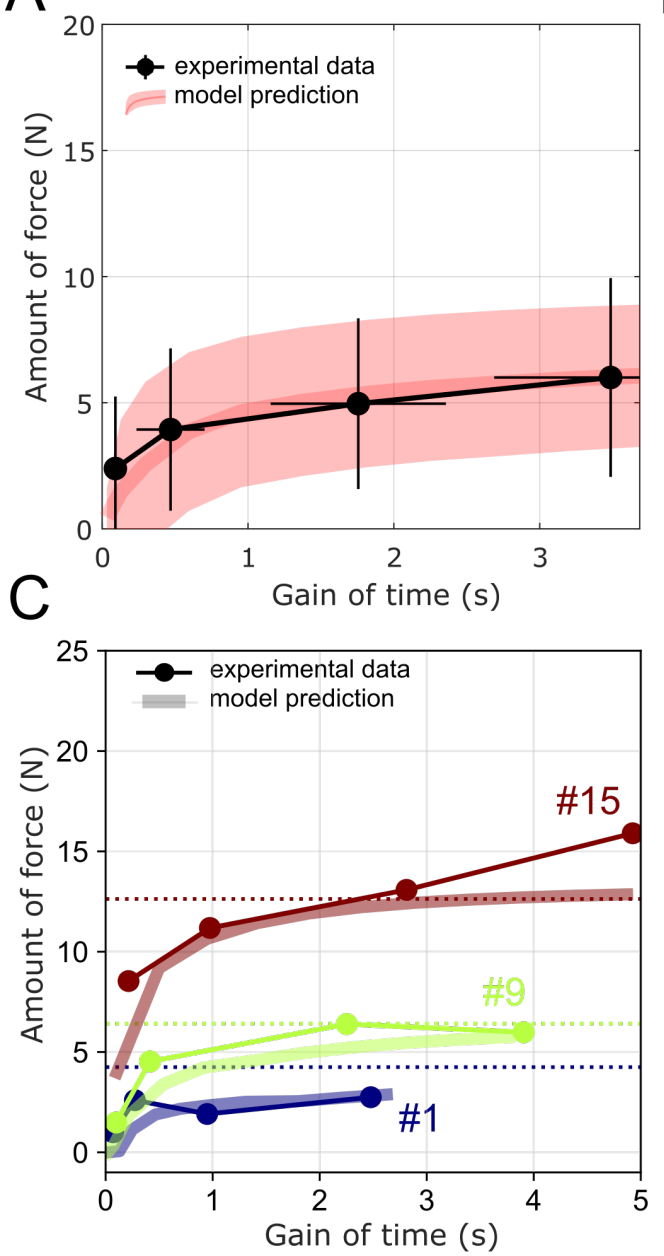

B
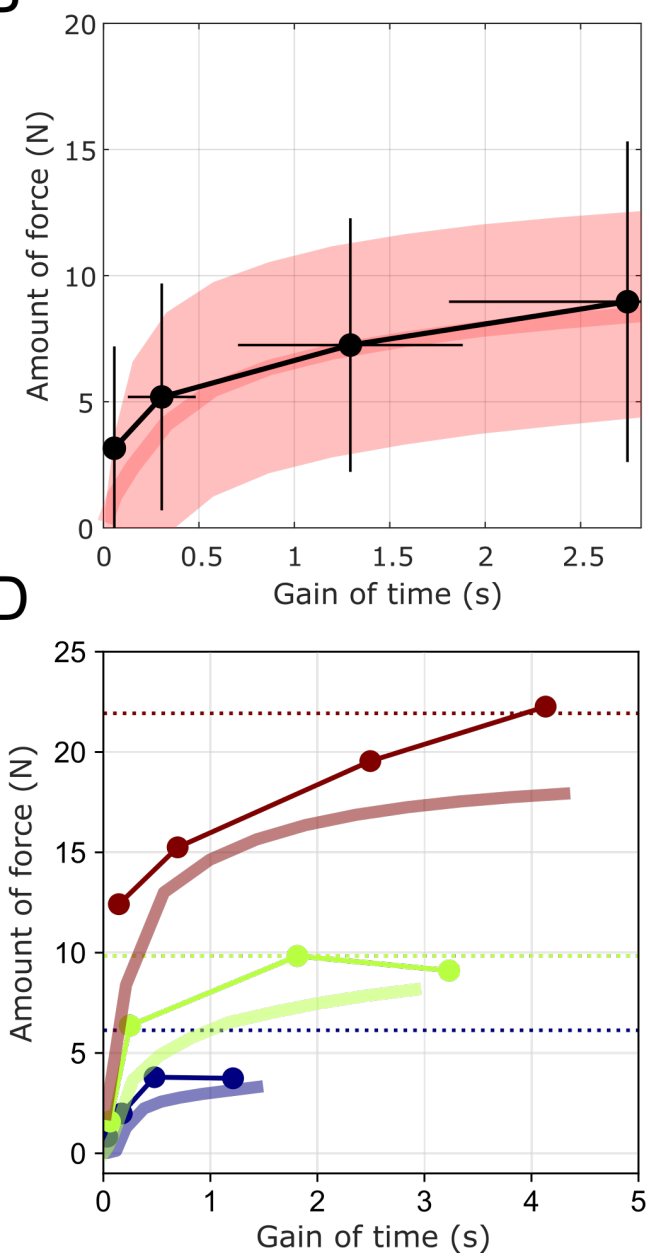

Figure 8: Relationship between gains of time and amount of forces used in Session 2. A. Mean experimental and simulated data for distance $0.2 \mathrm{~m}$. Black markers and traces are experimental data. The black filled circles correspond to the drives in the following order from left to right: $1 \mathrm{~s}$, $2 \mathrm{~s}, 4 \mathrm{~s}$ and $6 \mathrm{~s}$, respectively. Mean and standard deviations were calculated across participants. Red traces and shaded areas are model predictions (also mean and standard deviations across participants). The horizontal dotted line correspond to the mean amount of force measured in Session 1 for that distance. B. Same information for distance $0.4 \mathrm{~m}$. C-D. Same information for three participants with high, medium and low vigor respectively. 

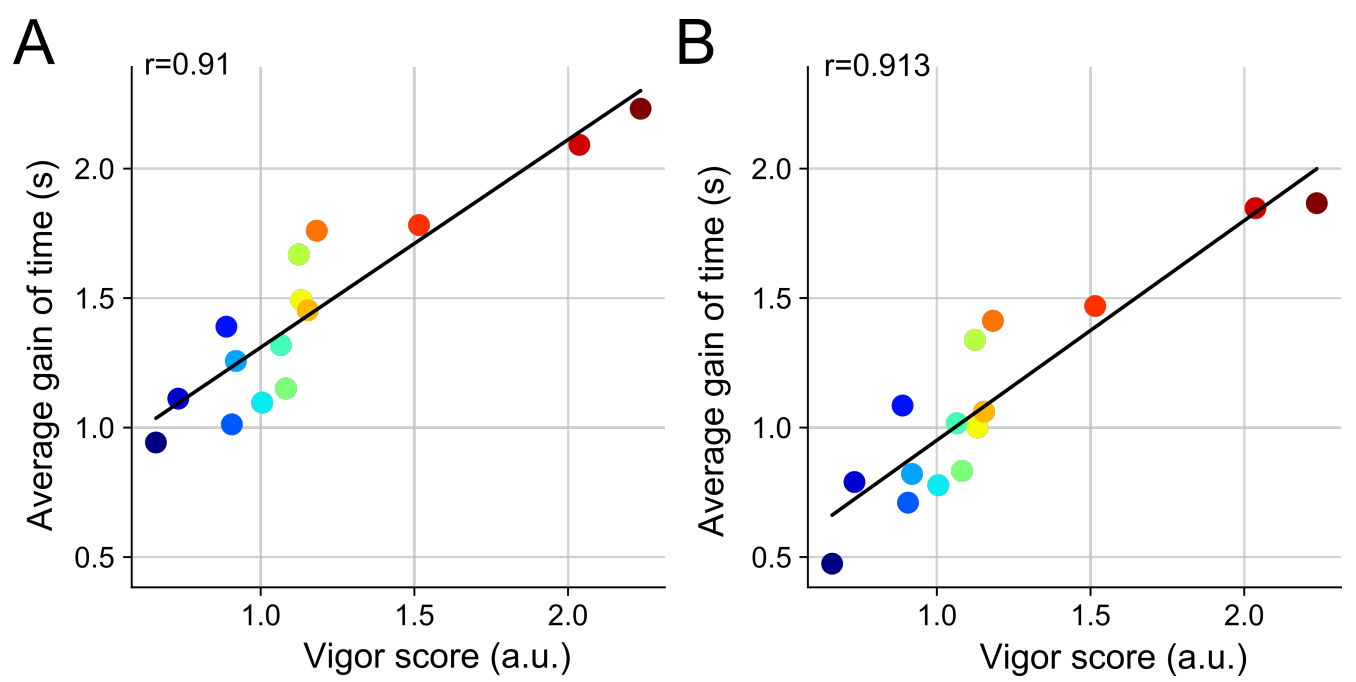

Figure 9: Relationship between vigor scores as measured in Session 1 and average gains of time in Session 2. Same color code than in Figure 5. Each dot is a participant. A. Distance $0.2 \mathrm{~m}$. B. Distance $0.4 \mathrm{~m}$.

unequivocally identify the cost of movement because of its potential compositeness and subjectivity (Berret et al., 2011; Summerside and Ahmed, 2021). The design of our task circumvents this issue by creating a condition in which the task accomplishes by itself, thereby neutralizing the cost of movement for specific motion durations. Despite this opportunity, our participants systematically exerted some force onto the joystick to achieve the task more quickly. They clearly favored saving time over saving energy in this task. Yet, when the drive was present, it was always optimal to remain inactive with respect to any cost of movement. This suggests that slow movements are generally avoided (van der Wel et al., 2010), not specifically because they may incur a large cost related to movement execution but mostly because they last long. This predominance of movement duration over energy expenditure was also found in a perceptual decision-making task at movement initiation (Lunazzi et al., 2021). Morel et al. (2017) have shown that duration is indeed a key factor that makes people judge a reaching movement as "effortful", and that this judgement does not seem to be based on objective measures such as metabolic energy. Here, it was clear that our participants did not minimize isometric force production alone, which suggests that the passage of time may contribute to action selection per se and perhaps to a subjective representation of effort. It has been proposed that the cost of time mainly expresses a subjective temporal discounting of reward within the brain, which could explain why the participant achieved the task more quickly than what is prescribed by the drive. This cost of time has been reviewed in several recent articles (Shadmehr et al., 2019; Carland et al., 2019) and a monograph (Shadmehr and Ahmed, 2020a), and might even reflect general personality traits (Choi et al., 2014; Berret et al., 2018). Hence the most vigorous participants should be the steepest temporal discounters and/or the ones who attribute the greatest subjective value to task accomplishment (Reppert et al., 2015; Yoon et al., 2020; Shadmehr and Ahmed, 2020a). This prediction agreed well with our findings that the most vigorous participants (as sorted from a baseline experiment) were the ones spending more energy to save a given amount of time. In our case, the cost of time may also capture an attentional cost related to the fact that participants were observing the motion of the cursor on the screen even when exerting no force, or just that they were engaged in the task. Since these higher-level costs are not strictly related to movement production processes, they are excluded from our definition of the cost of movement but can be integrated into a cognitive cost of time.

Although we mainly interpreted our results within the cost of time theory, it could be argued that participants just preferred to be active in the task. In this case, participants would also have preferred to move the cursor by themselves rather than to passively look at it. Assessing passivity from an amount of force lower than $0.15 \mathrm{~N}$, a total of $3,2,1$ and 0 participants passively executed the task for the drive durations $1 \mathrm{~s}, 2 \mathrm{~s}, 4 \mathrm{~s}$ and $6 \mathrm{~s}$ respectively (post-analysis for both distances). 
For instance, one participant (\#6) remained passive for drive durations $\leq 4$ s but suddenly became active when the drive lasted $6 \mathrm{~s}$. However, participants were generally active even for the smaller drive durations. The critical observation is that participants did not exert forces onto the joystick arbitrarily or just by habituation (e.g. using a fixed amount of force or fixed time in the task). In contrast, they saved time and exerted force in an idiosyncratic manner that was closely linked to their the original vigor in the task and that depended on the drive duration as predicted by an optimal control model based on their identified cost of time (which were obtained before the drive was introduced). Accordingly, highly vigorous participants were highly active in most conditions even for the $1 \mathrm{~s}$ or $2 \mathrm{~s}$ drives. These participants were clearly prone to spend a substantial effort to save a small amount of time. This may seem surprising (and it was not captured accurately by our model) but it fits well with the theory of vigor (Shadmehr and Ahmed, 2020a) and a putative link with personality traits like impulsivity or boredom proneness (Choi et al., 2014; Berret et al., 2018). Weakly vigorous participants were also active in most conditions but produced much lower amounts of force in the task, that is, they were also inclined to save time but much less than participants with high vigor scores. These observations show that participants did not simply prefer to be active with arbitrary levels of force and durations, nor did they try to replicate their baseline behaviour regardless of the duration of the drive. Instead, they energize the movement according to both their own vigor (or implicit motivation, Mazzoni et al., 2007) and the characteristics of the drive in agreement with an optimality principle minimizing a trade-off between a cost of movement and a cost of time. Another argument may be that the passage of time is subjectively faster when one is actively moving. The literature indeed suggests that the perception of time could be itself modulated by our ongoing voluntary actions (Eagleman, 2008; Merchant and Yarrow, 2016). For instance, saccadic eye movements seems to produce a time compression (Morrone et al., 2005), an observation which has been generalized to hand reaching movement and isometric force production (Tomassini et al., 2014). In the latter study, participants had to judge the time interval between tactile taps and time intervals defined by tactile stimuli were perceived as shorter when hand movements were prepared and executed. This psychophysical phenomenon is not incompatible with the hypothesis of a cognitive cost of time. Indeed, to either save true time or its subjective perception, it would have been worthwhile for the participants to energize task's achievement. Interestingly, the link between impulsivity and the sense of time have been reviewed in (Wittmann and Paulus, 2008) and it was suggested that "[impulsive] individuals are more likely to experience a slowing down of time during situations in which they are not able to act on their impulsive urges, for example when one has to wait for a delayed reward and is confronted with the passage of time".

To draw the present conclusions we relied on an isometric reaching task. The advantage of this task is that it allows resolving a number of issues that would occur with real movements. First, it removes the problem of dealing with participant-dependent or poorly known biomechanical limb parameters for different participants (inertia, centers of mass etc.). This lack of knowledge is typically problematic when estimating an objective cost of movement using musculoskeletal models. Here every participant controlled the same virtual point mass. Therefore, only the endpoint force was used to move the cursor, which allowed for a simpler definition of the movement cost in this task even though different choices were still possible. Here we used the amount of force as in (Shadmehr et al., 2016) (i.e. force-time integral) to estimate energy expenditure as objectively as possible from our experimental data. One limitation could be that we did not consider the metabolic cost of sitting with the arm still on the handle. However, this constant cost is unlikely to explain the motor decisions of the participants in our task as it is not related to movement but posture (see also Summerside and Ahmed, 2021 for a similar conclusion in a study estimating metabolic cost using expired gas analysis). Another limitation could be that we restricted our analysis to the force component on the y-axis. Additional principal component analyses however revealed that the horizontal force components linearly co-varied and that working with the norm of the force would only increase our estimation of effort by $1.3 \%$ on average, with idiosyncratic differences probably related to the specific posture of the participant in the task. Differences in vigor between participants could still arise from differences in their force production capacities, although this would not disprove the evidence for a cost of time in this task because the cost of movement was potentially neutralized in any case with the drive. Second, isometric reaching simplifies the implementation of the drive because there is no physical interaction between the 
moving cursor and the participant. It should be possible to create similar experimental tasks with real movements. For instance, generalization of this task could involve a long treadmill for walking or an exoskeleton/manipulandum for providing participants with assistive control laws at different speeds. An additional limitation of our study could be the choice to shift from the slowest to the fastest drives, but it was made to remove a potential confound. Indeed, practicing with a fast drive could have habituated the participant to execute the task more quickly than usual (Mazzoni et al., 2012). Consequently, the finding that a participant moved faster than their preferred speed in a slow-drive block (i.e. our premise) after having practiced extensively with a fast drive could have been attributed to some habituation effect and not to a cost of time. It is likely that this confound could be mitigated by considering many trials per block and analyzing the asymptotic behavior of the participant. Here we limited the number of trials per block to reduce other side-effects such as fatigue. Accordingly, we chose to start with slow-drive blocks to attribute any time saving to a cost of time and not to a strategy biased by previous practice with a quick drive. Yet, we did not test a fully randomized block-wise design and whether it would significantly change the behavior of the participants cannot be asserted. Finally, one could argue that an isometric reaching task is not ecological. Yet, isometric reaching tasks have been used successfully in the past to explore the neural control of movement, in particular trajectory planning, motor adaptation, muscle synergies and optimal control (Ghez et al., 1997; de Rugy et al., 2012, 2013; Berger et al., 2013; Rotella et al., 2013). Hence, it has proven to be an interesting paradigm that can provide relevant insights about the control of goal-directed actions. Choosing between time and effort is indeed a commonly encountered situation in daily life (e.g. the airport's treadmill example) and it is possible that the principles of such in-lab experiments will generalize to more ecological tasks such as choosing between taking the car or go by foot, take an escalator or climb the stairs, use his/her slow neuroprosthetic arm or healthy arm and so on. Future work will aim at using a motorized upper-limb exoskeleton to assist the movements of participants and test whether similar principles apply when muscles produce work and a real movement of the limb occurs. 


\section{${ }_{500}$ Acknowledgments}

${ }_{501}$ We thank Nicolò Balzarotti for his help during the data acquisition. We also thank Dorian Verdel ${ }_{502}$ and Jérémie Gaveau for useful comments on earlier versions of the manuscript. This work was 503 partly supported by the French National Agency for Research (grant ANR-19-CE33-0009). 


\section{References}

Berardelli, A., Rothwell, J. C., Thompson, P. D., and Hallett, M. (2001). Pathophysiology of bradykinesia in parkinson's disease. Brain, 124(Pt 11):2131-2146.

Berger, D. J., Gentner, R., Edmunds, T., Pai, D. K., and d'Avella, A. (2013). Differences in adaptation rates after virtual surgeries provide direct evidence for modularity. The Journal of neuroscience : the official journal of the Society for Neuroscience, 33:12384-12394.

Berret, B., Castanier, C., Bastide, S., and Deroche, T. (2018). Vigour of self-paced reaching movement: cost of time and individual traits. Sci. Rep., 8(1):1.

Berret, B., Chiovetto, E., Nori, F., and Pozzo, T. (2011). Evidence for composite cost functions in arm movement planning: an inverse optimal control approach. PLoS Comput. Biol., 7(10):e1002183.

Berret, B., Conessa, A., Schweighofer, N., and Burdet, E. (2021). Stochastic optimal feedforwardfeedback control determines timing and variability of arm movements with or without vision. PLOS Computational Biology, 17(6):e1009047.

Berret, B., Delis, I., Gaveau, J., and Jean, F. (2019). Optimality and Modularity in Human Movement: From Optimal Control to Muscle Synergies, pages 105-133. Springer International Publishing, Cham.

Berret, B. and Jean, F. (2016). Why don't we move slower? the value of time in the neural control of action. J. Neurosci., 36(4):1056-1070.

Berret, B. and Jean, F. (2020). Stochastic optimal open-loop control as a theory of force and impedance planning via muscle co-contraction. PLoS computational biology, 16:e1007414.

Carland, M. A., Thura, D., and Cisek, P. (2019). The urge to decide and act: Implications for brain function and dysfunction. The Neuroscientist, 25(5):491-511.

Choi, J. E. S., Vaswani, P. A., and Shadmehr, R. (2014). Vigor of movements and the cost of time in decision making. The Journal of neuroscience : the official journal of the Society for Neuroscience, 34:1212-1223.

de Rugy, A., Loeb, G. E., and Carroll, T. J. (2012). Muscle coordination is habitual rather than optimal. The Journal of neuroscience : the official journal of the Society for Neuroscience, 32:7384-7391.

de Rugy, A., Loeb, G. E., and Carroll, T. J. (2013). Are muscle synergies useful for neural control? Frontiers in computational neuroscience, 7:19.

Dudman, J. T. and Krakauer, J. W. (2016). The basal ganglia: from motor commands to the control of vigor. Curr. Opin. Neurobiol., 37:158-166.

Eagleman, D. M. (2008). Human time perception and its illusions. Current opinion in neurobiology, 18:131-136.

Engelbrecht, S. (2001). Minimum principles in motor control. J. Math. Psychol., 45(3):497-542.

Flash, T. and Hogan, N. (1985). The coordination of arm movements: an experimentally confirmed mathematical model. J. Neurosci., 5(7):1688-1703.

Gaveau, J., Berret, B., Demougeot, L., Fadiga, L., Pozzo, T., and Papaxanthis, C. (2014). Energyrelated optimal control accounts for gravitational load: comparing shoulder, elbow, and wrist rotations. J. Neurophysiol., 111(1):4-16.

Gaveau, J., Grospretre, S., Berret, B., Angelaki, D. E., and Papaxanthis, C. (2021). A cross-species neural integration of gravity for motor optimization. Science Advances, 7(15). 
Ghez, C., Favilla, M., Ghilardi, M. F., Gordon, J., Bermejo, R., and Pullman, S. (1997). Discrete and continuous planning of hand movements and isometric force trajectories. Experimental brain research, 115:217-233.

Guigon, E., Chafik, O., Jarrassé, N., and Roby-Brami, A. (2019). Experimental and theoretical study of velocity fluctuations during slow movements in humans. Journal of neurophysiology, 121:715-727.

Haith, A. M., Reppert, T. R., and Shadmehr, R. (2012). Evidence for hyperbolic temporal discounting of reward in control of movements. J. Neurosci., 32(34):11727-11736.

Harris, C. M. and Wolpert, D. M. (1998). Signal-dependent noise determines motor planning. Nature, 394(6695):780-784.

Hoff, B. (1994). A model of duration in normal and perturbed reaching movement. Biol. Cybern., pages $481-488$.

Huang, H. J. and Ahmed, A. A. (2012). Is there a reaching speed that minimizes metabolic cost? In Proceedings of the Translational and Computational Motor Control 2012, New Orleans, LA.

Labaune, O., Deroche, T., Teulier, C., and Berret, B. (2020). Vigor of reaching, walking, and gazing movements: on the consistency of interindividual differences. Journal of neurophysiology, 123:234-242.

Lenth, R. (2019). emmeans: Estimated Marginal Means, aka Least-Squares Means. R package version 1.4.2.

Liu, D. and Todorov, E. (2007). Evidence for the flexible sensorimotor strategies predicted by optimal feedback control. J. Neurosci., 27(35):9354-9368.

Lunazzi, C. S., Reynaud, A. J., and Thura, D. (2021). Dissociating the impact of movement time and energy costs on decision-making and action initiation in humans.

Manohar, S. G., Chong, T. T.-J., Apps, M. A. J., Batla, A., Stamelou, M., Jarman, P. R., Bhatia, K. P., and Husain, M. (2015). Reward pays the cost of noise reduction in motor and cognitive control. Current biology : CB, 25:1707-1716.

Mazzoni, P., Hristova, A., and Krakauer, J. W. (2007). Why don't we move faster? parkinson's disease, movement vigor, and implicit motivation. J. Neurosci., 27(27):7105-7116.

Mazzoni, P., Shabbott, B., and Cortés, J. C. (2012). Motor control abnormalities in parkinson's disease. Cold Spring Harbor perspectives in medicine, 2(6):a009282.

Merchant, H. and Yarrow, K. (2016). How the motor system both encodes and influences our sense of time. Current Opinion in Behavioral Sciences, 8:22-27.

Morel, P., Ulbrich, P., and Gail, A. (2017). What makes a reach movement effortful? physical effort discounting supports common minimization principles in decision making and motor control. PLoS Biol., 15(6):e2001323.

Morrone, M. C., Ross, J., and Burr, D. (2005). Saccadic eye movements cause compression of time as well as space. Nature neuroscience, 8:950-954.

Niv, Y., Daw, N. D., Joel, D., and Dayan, P. (2007). Tonic dopamine: opportunity costs and the control of response vigor. Psychopharmacology (Berl), 191(3):507-520.

Park, S.-W., Marino, H., Charles, S. K., Sternad, D., and Hogan, N. (2017). Moving slowly is hard for humans: Limitations of dynamic primitives. J. Neurophysiol., page jn.00643.2016.

Qian, N., Jiang, Y., Jiang, Z.-P., and Mazzoni, P. (2013). Movement duration, fitts's law, and an infinite-horizon optimal feedback control model for biological motor systems. Neural Comput., 25(3):697-724. 
R Core Team (2019). R: A Language and Environment for Statistical Computing. R Foundation for Statistical Computing, Vienna, Austria.

Ralston, H. J. (1958). Energy-speed relation and optimal speed during level walking. Int. Z. Angew. Physiol., 17(4):277-283.

Reppert, T. R., Lempert, K. M., Glimcher, P. W., and Shadmehr, R. (2015). Modulation of saccade vigor during value-based decision making. The Journal of neuroscience : the official journal of the Society for Neuroscience, 35:15369-15378.

Reppert, T. R., Rigas, I., Herzfeld, D. J., Sedaghat-Nejad, E., Komogortsev, O., and Shadmehr, R. (2018). Movement vigor as a traitlike attribute of individuality. J Neurophysiol, 120(2):741-757.

Rigoux, L. and Guigon, E. (2012). A model of reward- and effort-based optimal decision making and motor control. PLoS Comput. Biol., 8(10):e1002716.

Robbe, D. and Dudman, J. T. (2020). The Cognitive Neurosciences, chapter The Basal Ganglia Invigorate Actions and Decisions, pages 527-539. The Massachusetts Institute of Technology.

Rotella, M. F., Koehler, M., Nisky, I., Bastian, A. J., and Okamura, A. M. (2013). Adaptation to visuomotor rotation in isometric reaching is similar to movement adaptation. IEEE ... International Conference on Rehabilitation Robotics : [proceedings], 2013:6650431.

Shadmehr, R. (2010). Control of movements and temporal discounting of reward. Curr. Opin. Neurobiol., 20(6):726-730.

Shadmehr, R. and Ahmed, A. (2020a). Vigor: Neuroeconomics of Movement Control. MIT Press.

Shadmehr, R. and Ahmed, A. A. (2020b). Precis of vigor: Neuroeconomics of movement control. The Behavioral and Brain Sciences, pages 1-10.

Shadmehr, R., Huang, H. J., and Ahmed, A. A. (2016). A representation of effort in decisionmaking and motor control. Current biology : CB, 26:1929-1934.

Shadmehr, R., Orban de Xivry, J. J., Xu-Wilson, M., and Shih, T.-Y. (2010). Temporal discounting of reward and the cost of time in motor control. J. Neurosci., 30(31):10507-10516.

Shadmehr, R., Reppert, T. R., Summerside, E. M., Yoon, T., and Ahmed, A. A. (2019). Movement vigor as a reflection of subjective economic utility. Trends in neurosciences, 42:323-336.

Singmann, H., Bolker, B., Westfall, J., Aust, F., and Ben-Shachar, M. S. (2019). afex: Analysis of Factorial Experiments. R package version 0.25-1.

Stengel, R. (1986). Optimal Control and Estimation. Dover books on advanced mathematics. Dover Publications.

Summerside, E., Shadmehr, R., and Ahmed, A. A. (2018). Vigor of reaching movements: reward discounts the cost of effort. J. Neurophysiol.

Summerside, E. M. and Ahmed, A. A. (2021). Using metabolic energy to quantify the subjective value of physical effort. Journal of The Royal Society Interface, 18(180):20210387.

Takikawa, Y., Kawagoe, R., Itoh, H., Nakahara, H., and Hikosaka, O. (2002). Modulation of saccadic eye movements by predicted reward outcome. Exp. Brain Res., 142:284-291.

Thura, D. (2020). Decision urgency invigorates movement in humans. Behavioural Brain Research, $382: 112477$.

Todorov, E. (2004). Optimality principles in sensorimotor control. Nat. Neurosci., 7(9):907-915.

Todorov, E. and Jordan, M. I. (2002). Optimal feedback control as a theory of motor coordination. Nat. Neurosci., 5(11):1226-1235. 
Tomassini, A., Gori, M., Baud-Bovy, G., Sandini, G., and Morrone, M. C. (2014). Motor commands induce time compression for tactile stimuli. Journal of Neuroscience, 34(27):9164-9172.

Turner, R. S. and Desmurget, M. (2010). Basal ganglia contributions to motor control: a vigorous tutor. Curr. Opin. Neurobiol., 20(6):704-716.

Uno, Y., Kawato, M., and Suzuki, R. (1989). Formation and control of optimal trajectory in human multijoint arm movement. minimum torque-change model. Biol. Cybern., 61(2):89-101.

van Beers, R. J. (2008). Saccadic eye movements minimize the consequences of motor noise. PLoS One, 3(4):e2070.

van der Wel, R. P. R. D., Sternad, D., and Rosenbaum, D. A. (2010). Moving the arm at different rates: slow movements are avoided. Journal of motor behavior, 42:29-36.

Wang, C., Xiao, Y., Burdet, E., Gordon, J., and Schweighofer, N. (2016). The duration of reaching movement is longer than predicted by minimum variance. J. Neurophysiol., 116(5):2342-2345.

Wittmann, M. and Paulus, M. P. (2008). Decision making, impulsivity and time perception. Trends in cognitive sciences, 12(1):7-12.

Yoon, T., Geary, R. B., Ahmed, A. A., and Shadmehr, R. (2018). Control of movement vigor and decision making during foraging. Proceedings of the National Academy of Sciences of the United States of America, 115:E10476-E10485.

Yoon, T., Jaleel, A., Ahmed, A. A., and Shadmehr, R. (2020). Saccade vigor and the subjective economic value of visual stimuli. Journal of neurophysiology, 123:2161-2172.

Young, S. J., Pratt, J., and Chau, T. (2009). Target-directed movements at a comfortable pace: movement duration and fitts's law. J Mot Behav, 41(4):339-346. 